\title{
GROTHENDIECK TOPOLOGIES OVER COMPLETE LOCAL RINGS
}

\author{
BY STEPHEN S. SHATZ ${ }^{1}$
}

Communicated by A. Rosenberg, September 30, 1965

1. Introduction. J. Tate [8] has introduced a theory of cohomological dimension for fields using the êtale Grothendieck (=Galois) cohomology. In recent work, M. Artin has extended these methods to produce a dimension theory for noetherian preschemes. On the other hand, the author [5] has used the flat Grothendieck cohomology over a field to study certain duality questions (see also [7], [9] for the étale case); so it is natural to ask whether there exists a dimension theory based on the flat cohomology. We shall show that the answer is, in general, no. Full proofs will appear in [6].

2. Terminology. A Grothendieck topology is a pair consisting of a category Cat $T$ and a set Cov $T$ of families of morphisms of Cat $T$. They are subjected to the axioms:

(1) If $\phi$ is an isomorphism, $\{\phi\} \in \operatorname{Cov} T$.

(2) If $\left\{U_{i} \rightarrow U\right\} \in \operatorname{Cov} T$ and $\left\{V_{i j} \rightarrow U_{i}\right\} \in \operatorname{Cov} T$, for all $i$, then $\left\{V_{i j} \rightarrow U\right\} \in \operatorname{Cov} T$.

(3) If $\left\{U_{i} \rightarrow U\right\} \in \operatorname{Cov} T$ and $V \rightarrow U$ is arbitrary, then $U_{i} \times_{U} V$ exists for each $i$, and $\left\{U_{i} \times_{U} V \rightarrow V\right\} \in \operatorname{Cov} T$.

A presheaf (of abelian groups) on $T$ is a contravariant functor from Cat $T$ to the category of abelian groups, while a sheaf, $F$, is a presheaf which satisfies the axiom

$$
\begin{aligned}
& \text { For all }\left\{U_{i} \rightarrow U\right\} \in \operatorname{Cov} T, \text { the natural sequence } \\
& F(U) \rightarrow \prod_{i} F\left(U_{i}\right) \rightrightarrows \prod_{i, j} F\left(U_{i} \times_{U} U_{j}\right)
\end{aligned}
$$

is exact (i.e., $F(U)$ is mapped bijectively on to the set of all $x \in \prod_{i} F\left(U_{i}\right)$ whose images by the two maps shown agree in $\prod_{i, j} F\left(U_{i} \times_{U} U_{j}\right)$.) Roughly speaking, all that is done in Godement's book [2] for classical sheaf theory may be done in this general setting [1]. If $X$ is a prescheme [3, Vol. I, p. 97], we let Cat $T$ be the category of all preschemes $Y$ which are separated, finitely presented, flat, and quasifinite over $X$ [3, Vol. I, p. 135, p. 144; Vol. IV, p. 5; Vol. II, p. 115]. Cov $T$ consists of arbitrary families of flat morphisms whose disjoint sum is faithfully flat [3, Vol. IV, Part 2, p. 9]. It is known that these

\footnotetext{
${ }^{1}$ Supported in part by the National Science Foundation.
} 
data define a Grothendieck topology, which we call the flat topology on $X$.

\section{Main results.}

Theorem 1. Let $X=\operatorname{Spec} A$, with $A$ a complete, noetherian local ring of characteristic $p>0$. Unless $A$ is a perfect field, we have c.d.p $X=\infty$; that is, for every integer $n \geqq 0$, there exists a torsion sheaf ${ }^{2} F_{n}$ in the flat topology on $X$ such that the p-primary component of $H^{n}\left(X, F_{n}\right)$ is not zero. Moreover, if the residue field of $A$ is separably closed, then the sheaves $F_{n}($ for $n>0)$ may be chosen so that

$$
\begin{aligned}
& H^{r}\left(X, F_{n}\right)=(0) \text { for } r \neq 0, r \neq n \\
& H^{n}\left(X, F_{n}\right)=A^{+} / A^{+p} .
\end{aligned}
$$

Corollary. Let $k$ be a field of characteristic $p>0$. Then the following statements are equivalent:

(1) $k$ is perfect,

(2) $c . d \cdot p k \leqq 1$,

(3) c.d.p $k$ is finite,

(4) $c . d \cdot p k_{s}=0$,

(5) $c . d_{. p} k_{s}$ is finite.

Theorem 2. Let $k$ be a field of characteristic $p>0$. Let $G$ be a commutative group scheme [3, Vol. II, p. 166], [5, p. 412] of finite type over $k$. Then for every $r>2$ we have $H^{r}(k, G ; p)=(0)$. (Here, $H^{r}(X, F ; p)$ denotes the p-primary component of the group $H^{r}(X, F)$.) Consequently, by restricting the coefficient category to those sheaves which are representable (or their limits) we may bound the p-dimension of the field $k$ by 2.

4. Sketch of proofs. Over a complete local ring, one may replace quasi-finite by finite with no change in the dimension theory. Every finite algebra over $A$ is a complete semilocal ring, hence a direct product of complete local rings. Thus every object of Cat $T$ is uniquely a sum of connected schemes and all constructions and verifications may be restricted to the connected objects of Cat $T$. In the case of separably closed residue field, for each abelian group $a$ and each sheaf of sets $F$ over $X$, we define a presheaf $a_{F}$ by

$$
Q_{F}(U)=\coprod_{F(U)} \propto, \quad U \text { connected }
$$

where $\coprod_{F(U)} \propto$ means the direct sum of copies of $\propto$ indexed by the set $F(U)$, and we extend the definition of $Q_{F}$ in the usual way to the nonconnected objects of Cat $T$.

That is, a sheaf whose values lie in torsion abelian groups. 
Lemma. Let $A$ be as in Theorem 1 with separably closed residue field. Then the presheaves $Q_{F}$ are sheaves and for every object $U$ and covering $V$ of $U$ in Cat $T$,

$$
H^{r}\left(V / U, a_{F}\right)=(0) \text { for } r>0 .
$$

From equation $\left(^{* *}\right)$ one deduces that $H^{r}\left(U, a_{F}\right)=(0)$ for every $r>0$ and every object $U$ of Cat $T$. If $F_{1}=\mathfrak{a}_{p}$, where $\mathfrak{a}_{p}$ is the kernel of the Frobenius map on the additive group scheme $G_{a}$, then $F_{1}$ satisfies equation $\left({ }^{*}\right)$. The exact sequence

$$
0 \rightarrow F_{2} \rightarrow(Z / p Z)_{F_{1}} \rightarrow F_{1} \rightarrow 0
$$

and the above lemma, show that $F_{2}$ satisfies equation $\left({ }^{*}\right)$. One now proceeds by induction using the lemma and the exact sequence

$$
0 \rightarrow F_{n+1} \rightarrow(Z / p Z)_{F_{n}} \rightarrow F_{n} \rightarrow 0 .
$$

For the general case, one analyzes the Leray spectral sequence [1]

$$
H^{u}\left(X, R^{v} \pi_{*} F\right) \Rightarrow H^{*}\left(X_{s}, F\right)
$$

(where $X_{s}=\operatorname{Spec} A \otimes_{k} k_{s}, k=$ residue field of $A, k_{s}=$ separable closure of $k$ ).

The Corollary follows immediately from the theorem if one uses the Hochschild-Serre spectral sequence [1, p. 92] as applied to Grothendieck cohomology.

Theorem 2 is proved by reducing it to a question concerning artinian group schemes [5, pp. 412-413]. This is done via a structure theorem for the category of sheaves over $k$, and results of Tate. The conclusion is: in order to prove Theorem 2 it suffices to prove it for the kernel $G_{n}$ of the $n$th iterate of the Frobenius map on $G$. In this case, we make use of the Hochschild-Serre spectral sequence and the structure of a composition series for $G_{n}$ over $k_{s}$ to reduce the theorem to two assertions

(i) $H^{r}\left(k_{s}, \mathfrak{a}_{p}\right)=(0)$ for $r>1$,

(ii) $H^{r}\left(k_{s}, \mathfrak{u}_{p}\right)=(0)$ for $r>1$.

Here $\mathfrak{u}_{p}$ is the kernel of the Frobenius map on the multiplicative group scheme $\boldsymbol{G}_{m}$. Assertion (i) is known from [4, p. 21], [5, Proposition 3], and assertion (ii) follows because one can prove

$$
H^{r}\left(k_{8}, G_{m}\right)=(0) \text { for } r>0 .
$$

\section{REFERENCES}

1. M. Artin, Grothendieck topologies (mimeographed notes), Harvard University, Cambridge, Mass., 1962. 1958.

2. R. Godement, Topologie algêbrique et théorie des faisceaux, Hermann, Paris, 
3. A. Grothendieck and J. Dieudonné, Éléments degé ométrie algébrique. I-IV, Inst. Hautes Etudes Sci. 1960-1965.

4. A. Grothendieck, Techniques de descente et theorèmes d'existence en géomêtrie algébrique I: Généralités. Descente par morphismes fidelment plats, Séminaire Bourbaki, 1959-1960, Exposé 190, Paris.

5. S. Shatz, Cohomology of artinian group schemes over local fields, Ann. of Math. 79 (1964), 411-449.

6. - The cohomological dimension of certain Grothendieck topologies, Ann. of Math. (to appear).

7. J. Tate, Cohomology of abelian varieties over p-adic fields (cf. Notes by S. Lang), Princeton Univ., Princeton, N. J., May, 1959.

8. - Cohomology of profinite groups (cf. A. Douady, Cohomologie des groupes compacts totalements discontinus), Séminaire Bourbaki, 1959, Exposé 189, Paris.

9. —- Duality theorems in Galois cohomology over number fields, Proc. Stockholm Cong., August, 1962.

University of Pennsyluania 